'Unidad de Emergencia, Hospital Barros Luco Trudeau. Santiago, Chile. Internos de Medicina. Universidad Diego Portales. Santiago, Chile.

Trabajo no recibió financiamiento. Los autores declaran no tener conflictos de interés.

Recibido el 4 de junio de 2020, aceptado el 3 de septiembre de 2020.

Correspondencia a: Giancarlo Zuccone Z. gzucconez@gmail.com

Julio Matute Miranda juliocesarmatutemiranda@gmail. com

\section{Características clínicas y epidemiológicas del COVID-19 en la Unidad de Emergencia del Hospital Barros Luco: los primeros 164 pacientes}

\author{
GIANCARLO ZUCCONE, VALENTINA ALBORNOZa, \\ HELGA IBÁÑEZ ${ }^{\text {a }}$, RAÚL BETANCUR ${ }^{1}$, JULIO MATUTE ${ }^{1}$
}

\section{Features of 164 SARS-CoV-2 (+) patients consulting at an emergency room}

Background: The clinical manifestations, mortality rates, laboratory and imaging findings of SARS-CoV-2 infection are not yet fully elucidated. Aim: To describe the clinical manifestations, imaging and laboratory findings of 164 SARS-CoV-2 (+) patients consulting at an emergency room of a public general hospital in Santiago, Chile. Material and Methods: Review of medical records, imaging and laboratory results of 164 patients aged between 16 and 92 years (57.9\% males) consulting at the emergency room between March 12 and April 27, 2020. Results: The most common presenting symptom was dry cough in $47 \%$ of patients, followed by headache in $42 \%$ and myalgias in $42 \%$. Forty five percent referred contact with an infected patient or having travelled out of town. Thirty-three (20\%) required hospital admission and 5\% required admission to an intensive care unit. Conclusions: These patients were predominantly young, and their most common symptoms were dry cough, headache and myalgias.

(Rev Med Chile 2020; 148: 1096-1104)

Key words: Coronavirus; Emergency Service, Hospital; Severe Acute Respiratory Syndrome.

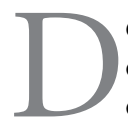
esde diciembre de 2019 se tiene registro de los primeros pacientes contagiados con un nuevo tipo de betacoronavirus envuelto ARN en Wuhan, China, denominado Severe Acute Respiratory Syndrome Corovairus 2 (SARS-CoV-2) $)^{1,8,9,18,19}$. Su presentación clínica sería variable, desde el portador asintomático hasta el paciente en estado crítico, con neumonía intersticial y SDRA grave $e^{1,3,7,11-13,16}$.

Se transmitiría por gotitas desde un paciente portador (al hablar, toser o estornudar) a uno no portador ${ }^{1,2,11-13,17,20}$. El virus sería viable un tiempo variable en distintas superficies, pudiendo adherirse a la piel del paciente previamente no portador, lo que explicaría los contagios sin contactos SARSCoV-2 (+) aparentes ${ }^{8,9,13,17,19}$.
Evidencia reciente postula que el virus tiene un período de incubación de hasta 14 días, desde su inoculación hasta la presentación de los síntomas, con una media de 4 a 5 días $^{11-14,17,19,20}$, y su riesgo de transmisión dependería de varios factores, entre ellos, el tiempo de exposición a un paciente sintomático o portador, la carga viral, las medidas de protección personal, uso de mascarilla en el paciente sintomático o sospechoso, mantención de la distancia social definida entre 1 y $1,5 \mathrm{~m}$ entre personas, hacinamiento o participación reciente en multitudes y exposición intrahospitalaria ${ }^{1,4,9,13,17-19}$.

Nuestro medio no es ajeno a la pandemia, teniendo casos confirmados desde inicios de marzo de 2020. Sin embargo, hay poca información publicada y disponible en nuestro medio acerca 
de la presentación clínica de los pacientes que consultan y sus comorbilidades, las alteraciones en los exámenes de laboratorio y otros datos, como características de los pacientes hospitalizados en sala o en la Unidad de Paciente Crítico (UPC).

Por lo anterior, en la Unidad de Emergencia del Hospital Barros Luco, y en conjunto con varias especialidades, se han elaborado diferentes pautas médicas, destacando la presentación de diferentes casos clínicos según sus antecedentes epidemiológicos y clínicos iniciales, esto con el fin de dar con el perfil de paciente que puede consultar en una urgencia de alta complejidad con enfermedad por SARS-CoV-2.

Presentamos este documento con la intención de aportar datos dada la coyuntura, según lo observado en nuestra Unidad de Emergencia, buscando características comunes entre los pacientes SARS-CoV-2 (+) que permitan establecer las presentaciones clínicas más prevalentes de la enfermedad.

\section{Materiales y Métodos}

Este estudio es retrospectivo descriptivo, buscando mostrar las características epidemiológicas y clínicas de los primeros pacientes SARS-CoV-2 $(+)$ en nuestro hospital.

La presente revisión se realizó en la Unidad de Emergencia (SU) del Hospital Barros Luco entre el 12 de marzo y el 27 de abril de 2020, logrando un $\mathrm{n}$ de 164 pacientes. Se revisaron todos los Datos de Atención de Urgencia (DAU) de los pacientes que fueron atendidos en el box COVID de la Unidad de Emergencia con PCR (+) para el virus. También se revisaron exámenes de laboratorio e imágenes que habían sido tomadas por protocolo interno según pauta médica del momento en la atención de pacientes sospechosos COVID. Dicha información fue almacenada y ordenada en una hoja Excel ${ }^{\circledR}$ para luego analizarse mediante porcentajes y medidas de tendencia central.

En paralelo, se realizó el seguimiento de los casos que se manejaron de forma ambulatoria a través de un equipo de salud liderado por un médico, vía llamados telefónicos y aplicando una encuesta para definir así los controles futuros de los pacientes SARS-CoV-2 (+) en manejo ambulatorio. En los hospitalizados, también se realizó seguimiento de parámetros clínicos, de laboratorio y de imágenes para tener así una mejor perspectiva de la evolución de los casos sin intervenir en el manejo y estudio médico respectivo a su complejidad.

El presente trabajo consta con aprobación del Comité de Ética Asistencial del Hospital Barros Luco según protocolo de calidad vigente.

\section{Resultados}

La muestra constó de 164 pacientes con PCR (+) para SARS-CoV-2, de los cuales $2(1,22 \%$ de la muestra) resultaron ser asintomáticos. Todo el resto (162 pacientes, 98,78\%) presentó síntomas y signos variados, a caracterizar más adelante.

\section{Características demográficas}

De esta muestra, 95 pacientes $(57,93 \%)$ correspondían al sexo masculino y $69(42,07 \%)$ al sexo femenino (Figura 1). El paciente de menor edad en consultar tenía 16 años, mientras que el mayor tenía 92. La edad promedio de estos pacientes SARS-CoV-2 (+) fue de 46 años, con una mediana de 45 años y una moda de 32. La distribución según rangos de edad de estos pacientes se detalla en la Tabla 1 y Figura 2.

Se presentan también los contagios diarios y acumulados de pacientes SARS-CoV-2 (+) en el intervalo de recogida de datos, destacando que $52 \%$ de los casos provenía de las comunas de San Miguel, Lo Espejo, Pedro Aguirre Cerda y San Joaquín, como se muestra en la Tabla 2 y Figura 3.

\section{Características clínicas}

La presentación clínica de los pacientes SARSCoV-2 fue variada, con combinaciones de sínto-

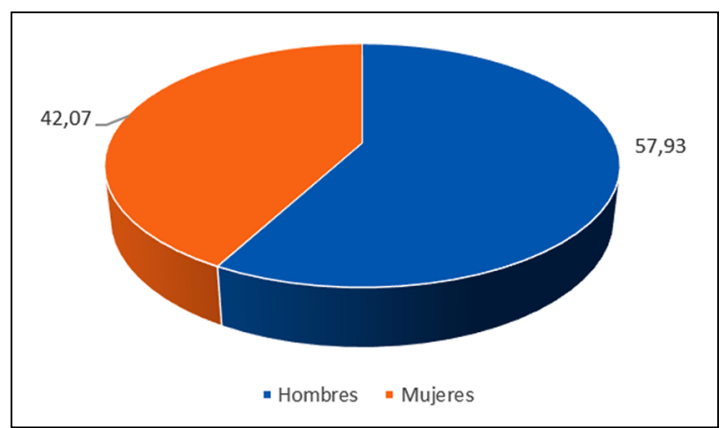

Figura 1. Distribución por sexo de los pacientes SARS-CoV-2 $(+)$. 
Tabla 1. Distribución por edad de los pacientes SARS-CoV-2 (+), con promedio, mediana y moda

\begin{tabular}{|c|c|c|}
\hline Rangos & Frecuencia & Porcentaje \\
\hline $15-19$ & 2 & 1,22 \\
\hline $20-24$ & 14 & 8,54 \\
\hline $25-29$ & 17 & 10,37 \\
\hline $30-34$ & 26 & 15,85 \\
\hline $35-39$ & 13 & 7,93 \\
\hline $40-44$ & 10 & 6,10 \\
\hline $45-49$ & 13 & 7,93 \\
\hline $50-54$ & 11 & 6,71 \\
\hline $55-59$ & 19 & 11,59 \\
\hline $60-64$ & 14 & 8,54 \\
\hline $65-69$ & 5 & 3,05 \\
\hline $70-74$ & 6 & 3,66 \\
\hline $75-79$ & 6 & 3,66 \\
\hline $80-84$ & 4 & 2,44 \\
\hline $85-89$ & 3 & 1,83 \\
\hline$>90$ & 1 & 0,61 \\
\hline Promedio & Mediana & Moda \\
\hline 46 & 45 & 32 \\
\hline
\end{tabular}

mas respiratorios y no respiratorios. El síntoma más frecuente fue la tos seca, referida en $46,95 \%$ de los casos ( 77 pacientes), seguido de cefalea en $42,07 \%$ de los casos (69 pacientes) y mialgias en $41,46 \%$ (68 pacientes). El detalle de los síntomas referidos y su frecuencia de presentación se ve en la Figura 4 y Tabla 3.

Por otro lado, 62 pacientes $(37,8 \%)$ refirieron tener alguna comorbilidad o factor de riesgo que propician a complicaciones asociadas a la infección por SARS-CoV-2 y que podrían requerir manejo hospitalario eventual, tales como: hipertensión arterial (HTA) y otros factores de riesgo cardiovascular, patología pulmonar previa o tabaquismo crónico activo o pasivo, diabetes mellitus, enfermedad renal crónica, obesidad y condiciones de inmunosupresión. Los otros 102 pacientes $(62,2 \%)$ no refirieron patologías de base o bien notificaron alguna comorbilidad que no corresponde al grupo de patologías mencionadas.

El tiempo promedio desde el inicio de los síntomas hasta la consulta en el SU fue de 4 días, con un tiempo mínimo de 1 día y un máximo de 21 días de síntomas previo a la consulta.

De esta muestra, 75 pacientes $(45,73 \%)$ refirieron haber tenido contacto con paciente SARS$\mathrm{CoV}-2(+)$ previo a la consulta o algún viaje fuera de la Región Metropolitana, pero dentro del país. Ninguno refirió viajes al extranjero. Los otros 89 pacientes $(54,27 \%)$ negaron contactos con pacientes positivos para SARS-CoV-2 o viajes.

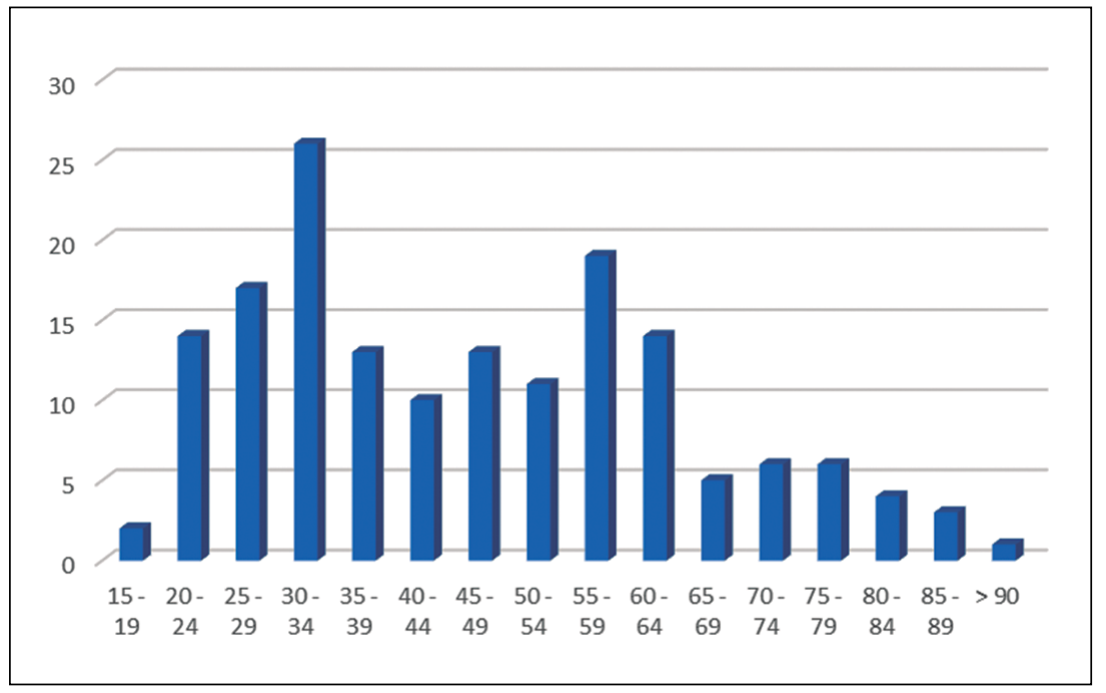

Figura 2. Distribución por grupos de edad de los pacientes SARS-CoV-2 (+). 
Tabla 2. Distribución porcentual de los pacientes SARS-CoV-2 (+) por comuna de residencia

\begin{tabular}{|lc|}
\hline Comuna & Porcentaje \\
\hline San Miguel & 16,15 \\
\hline Lo Espejo & 13,04 \\
\hline Pedro Aguirre Cerda & 11,8 \\
\hline San Joaquín & 11,8 \\
\hline La Cisterna & 8,7 \\
\hline Santiago & 8,7 \\
\hline San Bernardo & 6,83 \\
\hline El Bosque & 4,97 \\
\hline No Registrada & 3,73 \\
\hline Maipú & 1,86 \\
\hline Pudahuel & 1,86 \\
\hline Estación Central & 1,24 \\
\hline Quilicura & 1,24 \\
\hline Angol & 0,62 \\
\hline Buin & 0,62 \\
\hline Huechuraba & 0,62 \\
\hline lquique & 0,62 \\
\hline La Florida & 0,62 \\
\hline La Pintana & 0,62 \\
\hline Padre Hurtado & 0,62 \\
\hline Padre Las Casas & 0,62 \\
\hline Providencia & 0,62 \\
\hline Quinta Normal & 0,62 \\
\hline Renca & 0,62 \\
\hline San Ramón & 0,62 \\
\hline Talcahuano & 0,62 \\
\hline
\end{tabular}

De esta muestra, 122 pacientes $(74,39 \%)$ se encontraban en manejo domiciliario exclusivo o bien fueron dados de alta sin requerir manejo hospitalario posterior; 33 pacientes $(20,12 \%)$ requirieron manejo hospitalario en sala en algún momento de la evolución de la enfermedad y un total de 9 pacientes $(5,49 \%)$ requirieron manejo en UPC (Figura 5).

\section{Características imagenológicas}

Del total de pacientes, se tomaron exámenes de imagen (tomografía computada [TAC] de tórax) en 48 casos (29,3\% del total). De ellas, 39 presentaban patrón en vidrio esmerilado difuso uni o bilateral, concordantes con infección por SARS-CoV-2 (equivalente a $81,2 \%$ de los exámenes de imagen tomados), y 9 que no presentaban dichas alteraciones $(18,8 \%)$. Un ejemplo de las alteraciones descritas en 2 de estos pacientes se observa en la Figura $6^{21}$.

Del total de exámenes de imagen tomados, 38 (71,2\% del total de exámenes imagenológicos) corresponden a pacientes que requirieron tratamiento hospitalario en cama básica, intermedio o en Unidad de Cuidados Intensivos (UCI). De ellos, 35 mostraban alteraciones concordantes con infección por SARS-CoV-2 (92,1\%), 3 tenían imágenes sin alteraciones atribuibles a SARS-CoV-2 (7,9\%).

\section{Características de laboratorio}

En cuanto a los exámenes de laboratorio, comparamos entre pacientes de manejo ambulatorio y aquellos que requirieron manejo en

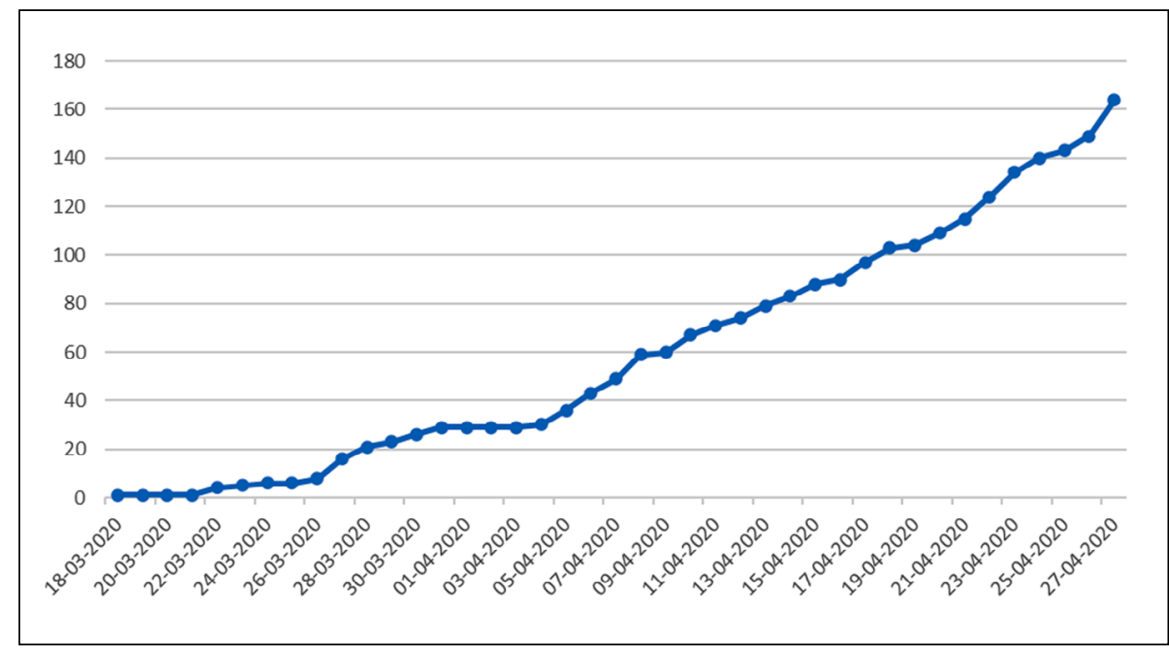

Figura 3. Curva de pacientes SARS-CoV-2 (+) acumulados por día hasta el 27-04-2020. 
Tabla 3. Recuento de pacientes SARS-CoV-2 (+) por día y acumulados al 27-04-2020

\begin{tabular}{|c|c|c|}
\hline Fecha & $\begin{array}{l}\text { Pctes. COVID(+) } \\
\text { acumulados }\end{array}$ & $\begin{array}{c}\text { Pctes. COVID } \\
(+) / \text { día }\end{array}$ \\
\hline $18-03-2020$ & 1 & 1 \\
\hline $19-03-2020$ & 1 & 0 \\
\hline 20-03-2020 & 1 & 0 \\
\hline 21-03-2020 & 1 & 0 \\
\hline $22-03-2020$ & 4 & 3 \\
\hline 23-03-2020 & 5 & 1 \\
\hline $24-03-2020$ & 6 & 1 \\
\hline $25-03-2020$ & 6 & 0 \\
\hline $26-03-2020$ & 8 & 2 \\
\hline $27-03-2020$ & 16 & 8 \\
\hline $28-03-2020$ & 21 & 5 \\
\hline $29-03-2020$ & 23 & 2 \\
\hline $30-03-2020$ & 26 & 3 \\
\hline $31-03-2020$ & 29 & 3 \\
\hline 01-04-2020 & 29 & 0 \\
\hline $02-04-2020$ & 29 & 0 \\
\hline 03-04-2020 & 29 & 0 \\
\hline 04-04-2020 & 30 & 1 \\
\hline 05-04-2020 & 36 & 6 \\
\hline 06-04-2020 & 43 & 7 \\
\hline 07-04-2020 & 49 & 6 \\
\hline 08-04-2020 & 59 & 10 \\
\hline 09-04-2020 & 60 & 1 \\
\hline $10-04-2020$ & 67 & 7 \\
\hline $11-04-2020$ & 71 & 4 \\
\hline $12-04-2020$ & 74 & 3 \\
\hline $13-04-2020$ & 79 & 5 \\
\hline $14-04-2020$ & 83 & 4 \\
\hline $15-04-2020$ & 88 & 5 \\
\hline $16-04-2020$ & 90 & 2 \\
\hline $17-04-2020$ & 97 & 7 \\
\hline $18-04-2020$ & 103 & 6 \\
\hline $19-04-2020$ & 104 & 1 \\
\hline $20-04-2020$ & 109 & 5 \\
\hline 21-04-2020 & 115 & 6 \\
\hline $22-04-2020$ & 124 & 9 \\
\hline 23-04-2020 & 134 & 10 \\
\hline 24-04-2020 & 140 & 6 \\
\hline $25-04-2020$ & 143 & 3 \\
\hline 26-04-2020 & 149 & 6 \\
\hline 27-04-2020 & 164 & 15 \\
\hline
\end{tabular}

UCI, los valores de proteína $\mathrm{C}$ reactiva (PCR) y de ferritina como marcadores inflamatorios, troponina T como marcador de daño miocárdico, deshidrogenasa láctica (LDH) en correlación con daño pulmonar y de dímero D (DD), como marcador de trastorno de la coagulación, utilizando promedio y mediana como medidas de tendencia central. Su comparación tanto en promedio como en mediana se representan en la Figura 7.

\section{a. Dímero $D$}

El valor máximo para el DD registrado en los pacientes en manejo ambulatorio fue de 1.811 $\mu \mathrm{g} / \mathrm{L}$, con un promedio de $574 \mu \mathrm{g} / \mathrm{L}$ y una mediana de $484 \mu \mathrm{g} / \mathrm{L}$. Esto versus los pacientes manejados en UCI, con un valor máximo registrado de 6.558 $\mu \mathrm{g} / \mathrm{L}$, un promedio de $2.791 \mu \mathrm{g} / \mathrm{L}$ y una mediana de $1.826 \mu \mathrm{g} / \mathrm{L}$.

\section{b. Ferritina}

El valor máximo para ferritina registrado en los pacientes en manejo ambulatorio fue de 1.935 $\mathrm{ng} / \mathrm{mL}$, con un promedio de $540 \mathrm{ng} / \mathrm{mL}$ y una mediana de $315 \mathrm{ng} / \mathrm{mL}$. En los pacientes manejados en UCI, el valor máximo registrado fue de 5.190 $\mathrm{ng} / \mathrm{mL}$, con un promedio de $1.925 \mathrm{ng} / \mathrm{mL}$ y una mediana de $1.776 \mathrm{ng} / \mathrm{mL}$.

\section{c. $L D H$}

El valor máximo para la $\mathrm{LDH}$ en los pacientes ambulatorios fue de $421 \mathrm{U} / \mathrm{L}$, con un promedio de 264 U/L y una media de 259 U/L. En los pacientes manejados en UCI, el máximo registrado fue de $648 \mathrm{U} / \mathrm{L}$, con un promedio de $440 \mathrm{U} / \mathrm{L}$ y una mediana de $441 \mathrm{U} / \mathrm{L}$.

\section{d. Troponina $T$}

El valor máximo para la troponina $\mathrm{T}$ en los pacientes en manejo ambulatorio fue de $283 \mathrm{ng} / \mathrm{L}$, con un promedio de $35 \mathrm{ng} / \mathrm{L}$ y una mediana de $7 \mathrm{ng} / \mathrm{L}$. En los pacientes manejados en UCI, el máximo fue de $234 \mathrm{ng} / \mathrm{L}$, un promedio de $58 \mathrm{ng} / \mathrm{L}$ y una mediana de $22 \mathrm{ng} / \mathrm{L}$.

e. PCR

El valor máximo de PCR en los pacientes ambulatorios fue de $100,6 \mathrm{mg} / \mathrm{L}$, con un promedio de $34 \mathrm{mg} / \mathrm{L}$ y una media de $18 \mathrm{mg} / \mathrm{L}$ versus los pacientes manejados en UCI, con un valor máximo de $550 \mathrm{mg} / \mathrm{L}$, un promedio de $182 \mathrm{mg} / \mathrm{L}$ y una mediana de $152 \mathrm{mg} / \mathrm{L}$. 


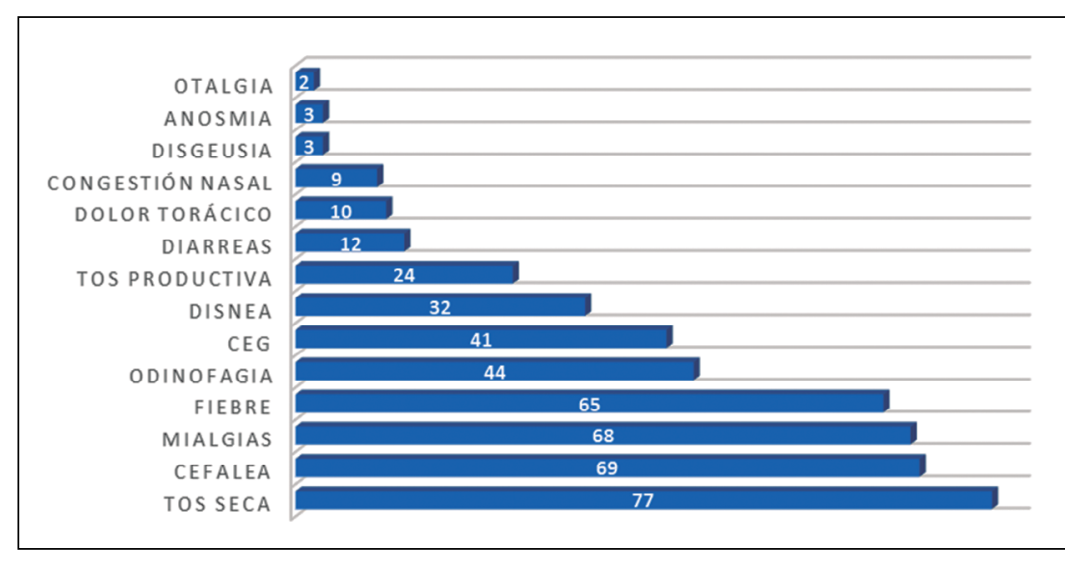

Figura 4. Frecuencia de presentación de los síntomas referidos por los pacientes SARS-CoV-2 (+) al momento de consultar en la Unidad de Emergencia.
Tabla 4. Frecuencia de presentación y porcentaje de los síntomas referidos por los pacientes SARS-CoV-2 (+) al momento de consultar en la Unidad de Emergencia

\begin{tabular}{|lcc|}
\hline Síntomas & $\begin{array}{c}\text { Frecuencia de } \\
\text { presentación }\end{array}$ & Porcentaje \\
\hline Tos seca & 77 & 46,95 \\
\hline Cefalea & 69 & 42,07 \\
\hline Mialgias & 68 & 41,46 \\
\hline Fiebre & 65 & 39,63 \\
\hline Odinofagia & 44 & 26,83 \\
\hline CEG & 41 & 25,00 \\
\hline Disnea & 32 & 19,51 \\
\hline Tos productiva & 24 & 14,63 \\
\hline Diarreas & 12 & 7,32 \\
\hline Dolor torácico & 10 & 6,10 \\
\hline Congestión nasal & 9 & 5,49 \\
\hline Disgeusia & 3 & 1,83 \\
\hline Anosmia & 3 & 1,83 \\
\hline Otalgia & 2 & 1,22 \\
\hline & & \\
\hline
\end{tabular}

\section{Discusión}

Este trabajo pretende ser meramente descriptivo, por lo que sus resultados podrían ser extrapolables a la realidad de otras Unidades de Emergencia de alta complejidad a nivel nacional. Actualmente sabemos que hay un porcentaje no despreciable de

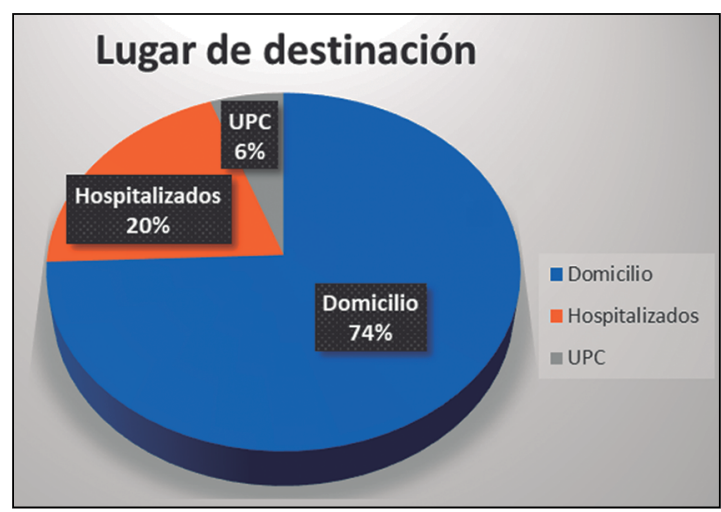

Figura 5. Porcentaje de pacientes SARS-CoV-2 (+) según lugar de manejo.

portadores asintomáticos con capacidad de contagiar $^{1,3,5,9,10,13,17,19}$. En su mayoría, los pacientes que consultaron con síntomas de SARS-CoV-2 fueron hombres. La distribución por edad de los pacientes que consultaron fue bastante amplia, con su promedio en torno a los 45 años y un mayor número en el grupo entre los 30 y los 34 años, y existe una tendencia a no reportar un contacto conocido positivo para SARS-CoV-2 previo al diagnóstico por PCR. Todo lo anterior podría tener que ver con contextos laborales de cada paciente, en los cuales quizás no existía o no fue posible implementar medidas como el teletrabajo, en asociación a otras condiciones desfavorables, como uso obligado del transporte público, necesidad de trabajo o abastecimiento en lugares de alta concentración de gente como lo son ferias libres, y condiciones de 


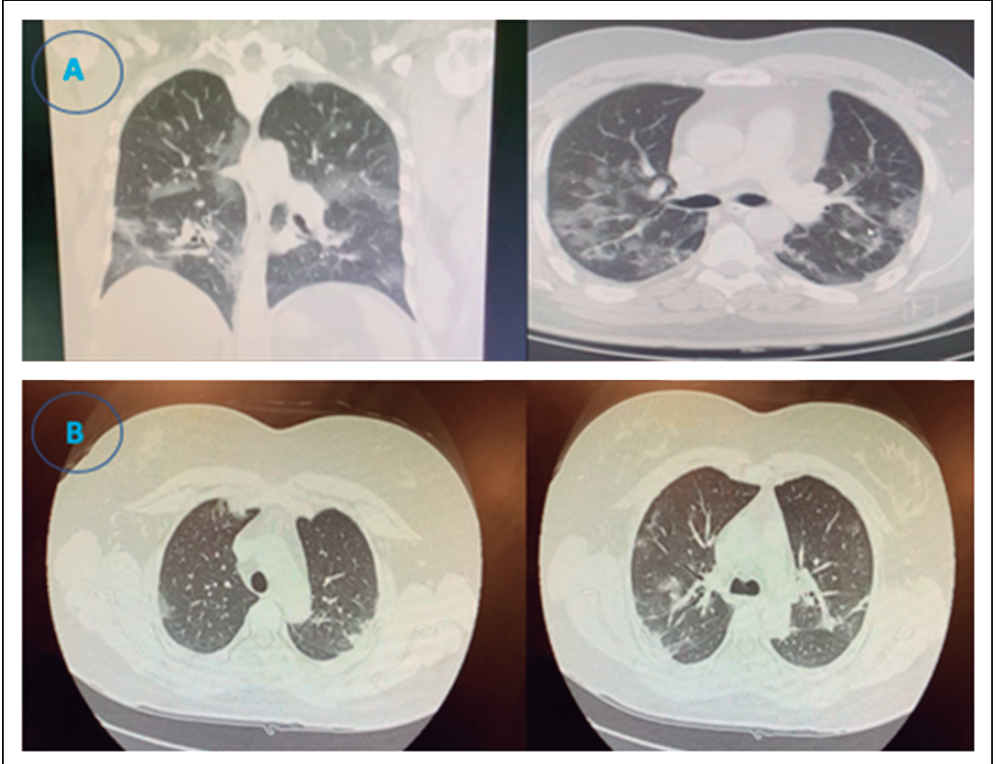

Figura 6. Características imagenológicas del COVID-19: opacidades en vidrio esmerilado, consolidaciones y patrón en pavimento empedrado o paving crazy. A) Imágenes iniciales de un paciente que queda hospitalizado en UPC con necesidad de VMI a las $36 \mathrm{~h}$ de su ingreso. B) Imágenes iniciales de un paciente con manejo y seguimiento ambulatorio con buena evolución clínica.
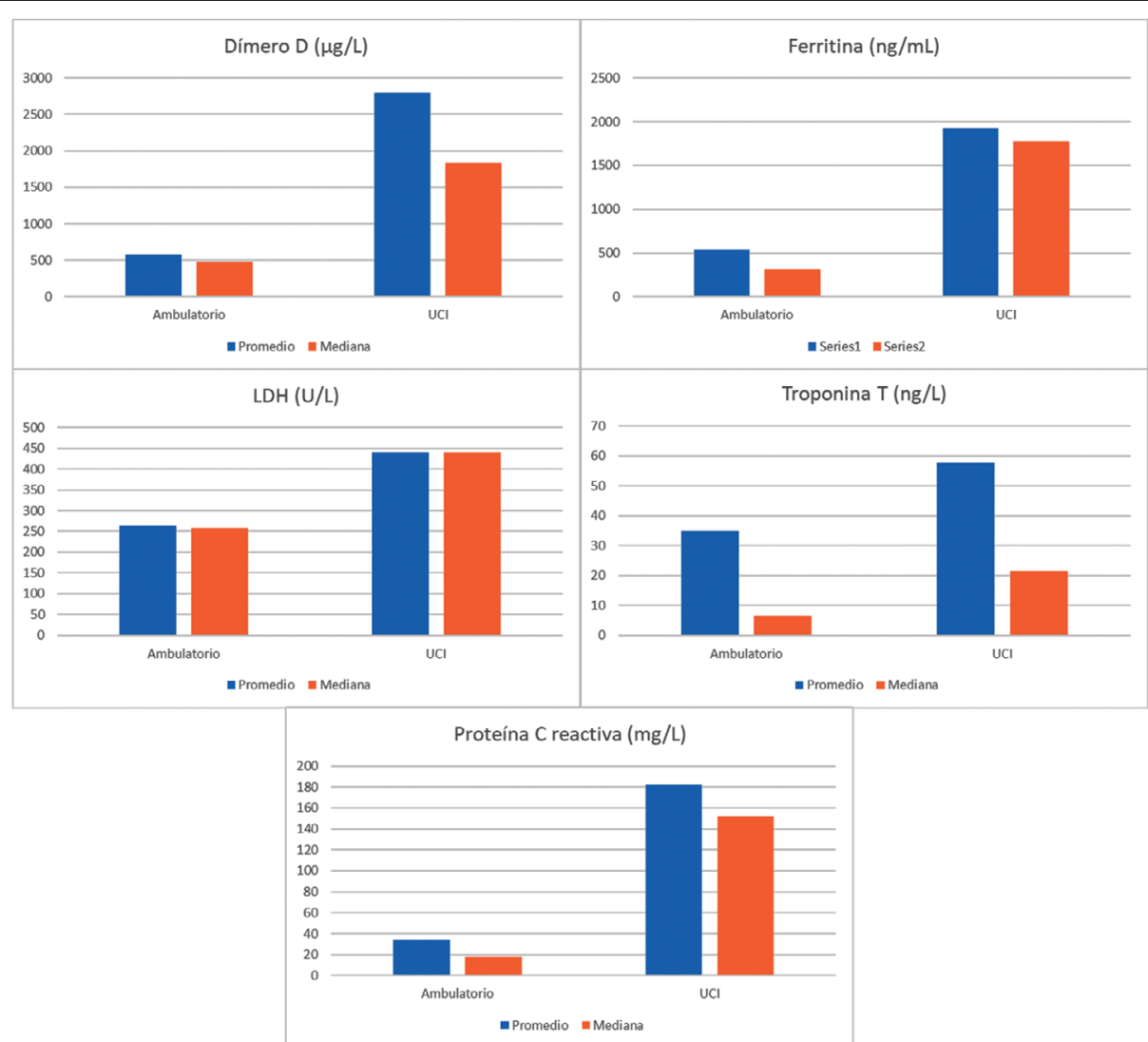

Figura 7. Comparación de distintos parámetros de laboratorio en pacientes ambulatorios (columnas izquierdas) y en pacientes $\mathrm{UCl}$ (columnas derechas). En azul se ven los valores promedio y en naranjo los valores de mediana. 
vivienda que no permiten el aislamiento efectivo entre sus habitantes, como lo serían habitaciones compartidas y disponibilidad limitada de baños exclusivos para gente contagiada ${ }^{3,4,8,17,19}$. La pérdida en la trazabilidad de la cadena de contagio podría explicarse tanto por los portadores asintomáticos con capacidad de contagio en asociación a los factores de aglomeración de gente ya descritos ${ }^{2,4,11,17,19}$.

La curva de contagio en esta etapa inicial de la pandemia muestra, en su primera fase, una característica en apariencia lineal, con un quiebre en su pendiente y al alza en torno al día 5 de abril. El tiempo promedio de presencia de síntomas fue de casi 4 días antes de consultar, compatible con otros reportes similares ${ }^{4,8,9,11-13,15,18-20}$.

En cuanto a los exámenes de imágenes y laboratorio, parece haber cierta correlación entre la gravedad del paciente (definida por su nivel de manejo, si fue en domicilio o en contexto hospitalario $\mathrm{y}$, en este último, en sala versus $\mathrm{UCI})^{16,19}$, las alteraciones imagenológicas y los valores y alteraciones de laboratorio presentes ${ }^{16,21}$. La presencia de ferritina y PCR altas, ambos marcadores inflamatorios, fue creciendo en la medida en que fue requerido manejo médico más especializado ${ }^{16,19}$. Lo mismo ocurre con la presencia de alteraciones en otros parámetros, sobre todo el dímero $\mathrm{D}$ y troponina $\mathrm{T}$, lo que se podría traducir en un mayor compromiso de la coagulación y miocárdico en los pacientes más graves $^{16,19}$. La elevación de estos parámetros fue más común y marcada conforme se avanzaba en el nivel de manejo (desde domicilio a UCI), y la evidencia disponible los cita como marcadores de gravedad y mal pronóstico, concordante con lo visto en esta muestra $3,5,9,13,16,19$.

Entre las limitaciones de este documento se cuentan el hecho de que los datos obtenidos fueron todos desde recopilaciones posteriores al momento de la consulta, mediante lectura de los DAU y registro del sistema de exámenes solicitados, y si el médico que realizó la atención no registró los hallazgos en el DAU, estos no fueron incluidos en el estudio. Asimismo, si bien siempre se intentó corroborar y confirmar otros datos al momento de notificar, sobre todo el domicilio de los pacientes contagiados, sí nos encontramos con datos no concordantes que pudiesen incidir en la calidad de este registro.

El número de pacientes en el presente estudio observacional es de nivel medio dado el contexto inicial de la pandemia en el país, pero parecido al n que se publica en la mayoría de los trabajos recientes. La comparación con otros estudios de esta índole, con mayor $\mathrm{n}$ de pacientes y análisis estadístico, se hace necesaria para la construcción de evidencia clínica más sólida.

Dentro de las ventajas, se cuenta que la metodología de obtención de datos es altamente replicable en otros centros, con parámetros objetivos y subjetivos bien definidos, lo cual da pie a que se escriban otros trabajos similares que faciliten el análisis estadístico global de la pandemia a nivel de los distintos centros hospitalarios a nivel nacional. La posibilidad de acceso tanto a los DAU, exámenes de laboratorio y de imagen de manera retrospectiva, en conjunto con la rápida confirmación del diagnóstico de los pacientes SARS-CoV-2 $(+)$ en las etapas iniciales de la pandemia, permitió que el desarrollo de este trabajo se diera de forma rápida y fluida a pesar del estado de emergencia sanitaria.

\section{Conclusiones}

Como resultado final, tenemos que el paciente con COVID-19 que consultó en nuestra Unidad de Emergencia se caracterizó por ser mayoritariamente joven, de sexo masculino, en los que destacaron la tos no productiva, cefalea y mialgias como síntomas de presentación más frecuentes, $y$ fue más prevalente que los pacientes SARS-CoV-2 $(+)$ no tuviesen conocimiento certero de un contacto positivo con otro portador del virus.

Buena parte de esta muestra requirió manejo solo en domicilio, aunque un porcentaje no despreciable hizo complicaciones lo suficientemente graves como para requerir hospitalización en UCI.

Parámetros sanguíneos como la ferritina, PCR, dímero $\mathrm{D}$ y troponina $\mathrm{T}$ son los que parecen alterarse en mayor medida en los pacientes cursando cuadros más graves de COVID-19, por lo que creemos pudiesen ser marcadores pronósticos importantes en los pacientes con enfermedad por SARS-CoV-2 sintomática. Asimismo, creemos que hay que hacer seguimiento estricto de aquellos pacientes cuya clínica no amerita hospitalización al momento de consultar en el servicio de urgencia, pero que cuenten con alguno de los factores de riesgo expuestos o alteraciones en los exámenes de laboratorio como las descritas. 


\section{Referencias}

1. Guan WJ, Ni ZY, Hu Y, Liang WH, Ou CQ, He JX, et al. Clinical characteristics of coronavirus disease 2019 in China. N Engl J Med 2020; 382: 1708-20.

2. Zhu N, Zhang D, Wang W, Li X, Yang B, Song J, et al. A Novel Coronavirus from Patients with Pneumonia in China, 2019. N Engl J Med 2020; 382: 727-33.

3. Su S, Wong G, Shi W, Liu J, Lai ACK, Zhou J, et al. Epidemiology, genetic recombination, and pathogenesis of coronaviruses. Trends Microbiol 2016; 24: 490-502.

4. She J, Jiang J, Ye L, Hu L, Bai C, Song Y. 2019 novel coronavirus of pneumonia in Wuhan, China: emerging attack and management strategies. Clin Trans Med 2020; 9: 19.

5. Weiss SR, Leibowitz JL. Coronavirus pathogenesis. Adv Virus Res 2011; 81: 85-164.

6. Huang C, Wang Y, Li X, Ren L, Zhao J, Hu Y, et al. Clinical features of patients infected with 2019 novel coronavirus in Wuhan, China. Lancet 2020; 395 (10223): 497-506.

7. Wang D, Hu B, Hu C, Zhu F, Liu X, Zhang J, et al. Clinical Characteristics of 138 Hospitalized Patients With 2019 Novel Coronavirus-Infected Pneumonia in Wuhan, China. JAMA 2020; 323 (11): 1061-9.

8. Chang D, Lin M, Wei L, Xie L, Zhu G, Dela Cruz CS, et al. Epidemiologic and Clinical Characteristics of Novel Coronavirus Infections Involving 13 Patients Outside Wuhan, China. JAMA 2020; 323 (11): 1092-3.

9. McIntosh K. Coronavirus Disease 2019 (COVID-19): Epidemiology, clinical features, diagnosis, and prevention. UpToDate, 2020.

10. Xu XW, Wu XX, Jiang XG, Xu KJ, Ying LJ, Ma CL, et al. Clinical findings in a group of patients infected with the 2019 novel coronavirus (SARS-CoV-2) outside of Wuhan, China: retrospective case series. BMJ 2020; 368 : m606.

11. Chen N, Zhou M, Dong X, Qu J, Gong F, Han Y, et al. Epidemiological and clinical characteristics of 99 cases of 2019 novel coronavirus pneumonia in Wuhan, China: a descriptive study. Lancet 2020; 395 (10223): 507-13.

12. Zheng Y, Xiong C, Liu Y, Qian X, Tang Y, Liu L, et al. Epidemiological and clinical characteristics analysis of COVID-19 in surrounding areas of Wuhan, Hubei Province in 2020. Pharmacol Res 2020; 157: 104821.

13. Ge H, Wang X, Yuan X, Xiao G, Wang C, Deng T, et al. The epidemiology and clinical information about COVID-19. Eur J Clin Microbiol Infect Dis 2020; 39 (6): 1011-9.

14. Masse S, Capai L, Villechenaud N, Blanchon T, Charrel R, Falchi A. Epidemiology and clinical symptoms related to seasonal coronavirus identified in patients with acute respiratory infections consulting in primary care over six influenza seasons (2014-2020) en France. Viruses 2020; 12 (6): 630 .

15. Zhang JJ, Dong X, Cao YY, Yuan YD, Yang YB, Yan YQ, et al. Clinical characteristics of 140 patients infected with SARS-CoV-2 in Wuhan, China. Allergy 2020; 75 (7): 1730-41.

16. Yang X, Yu Y, Xu J, Shu H, Xia J, Liu H, et al. Clinical course and outcomes of critically ill patients with SARSCoV-2 pneumonia in Wuhan, China: a single-centered, retrospective, observational study. Lancet 2020; 8 (5): 475-81.

17. Rothe C, Schunk M, Sothmann P, Bretzel G, Froeschl G, Wallrauch C, et al. Transmission of 2019-nCoV infection from an asymptomatic contact in Germany. N Engl J Med 2020; 382; 10.

18. Carlos WG, Dela Cruz CS, Cao B, Pasnick S, Jamil S. Novel Wuhan (2019-nCoV) Coronavirus. Am J Respir Crit Care Med 2020; 201 (4): 7-8.

19. Singhal T. A review of coronavirus disease-2019 (COVID-19). Indian J Pediatr 2020; 87 (4): 281-6.

20. Holshue ML, DeBolt C, Lindquist S, Lofy KH, Wiesman J, Bruce H, et al. First case of 2019 novel coronavirus in the United States. N Engl J Med 2020; 382: 10.

21. Wang H, Wei R, Rao G, Zhu J, Song B. Characteristic CT findings distinguishing 2019 novel coronavirus disease (COVID-19) from influenza pneumonia. Eur Radiol 2020; 30 (9): 4910-7. 